COCHRANE CORNER

${ }^{\dagger}$ This review is an abridged version of a Cochrane review previously published in the Cochrane Database of Systematic Reviews, 2017, July 5, Issue 7: CD011520 (doi: 10.1002/ 14651858.CD011520.pub2) (see www.Cochranelibrary.com for information). Cochrane reviews are regularly updated as new evidence emerges and in response to feedback, and the Cochrane Database of Systematic Reviews should be consulted for the most recent version of the review.

Copyright (C) 2018 The Cochrane Collaboration. Published by John Wiley \& Sons Ltd.

We thank the Cochrane Review Group for their support in publishing these reviews.

\title{
Vortioxetine for depression in adults ${ }^{\dagger}$
}

Markus Koesters, Giovanni Ostuzzi, Giuseppe Guaiana, Johanna Breilmann \& Corrado Barbui

\section{Background}

Major depressive disorder is a common mental disorder affecting a person's mind, behaviour and body. It is expressed as a variety of symptoms and is associated with substantial impairment. Despite a range of pharmacological and non-pharmacological treatment options, there is still room for improvement of the pharmacological treatment of depression in terms of efficacy and tolerability. The latest available antidepressant is vortioxetine. It is assumed that vortioxetine's antidepressant action is related to a direct modulation of serotonergic receptor activity and inhibition of the serotonin transporter. The mechanism of action is not fully understood, but it is claimed to be novel. Vortioxetine was placed in the category of 'Other' antidepressants and may therefore provide an alternative to existing antidepressant drugs.

\section{Objectives}

To assess the efficacy and acceptability of vortioxetine compared with placebo and other antidepressant drugs in the treatment of acute depression in adults.

Search methods

We searched Cochrane's Depression, Anxiety and Neurosis Review Group's Specialised Register to May 2016 without applying any restrictions to date, language or publication status. We checked reference lists of relevant studies and reviews, regulatory agency reports and trial databases.

\section{Selection criteria}

We included randomised controlled trials comparing the efficacy, tolerability or both of vortioxetine versus placebo or any other antidepressant agent in the treatment of acute depression in adults.

\section{Data collection and analysis}

Two review authors independently selected the studies and extracted data. We extracted data on study characteristics, participant characteristics, intervention details and outcome measures in terms of efficacy, acceptability and tolerability. We analysed intention-to-treat (ITT) data only and used risk ratios (RR) as effect sizes for dichotomous data and mean differences (MD) for continuous data, with 95\% Cl. Meta-analyses used random-effects models.

\section{Main results}

We included 15 studies (7746 participants) in this review. Seven studies were placebo controlled; eight studies compared vortioxetine with serotonin-noradrenaline reuptake inhibitors (SNRIs). We were unable to identify any study that compared vortioxetine with antidepressant drugs from other classes, such as selective serotonin reuptake inhibitors (SSRIs).

Vortioxetine may be more effective than placebo across the three efficacy outcomes: response (Mantel-Haenszel RR $=1.35$, 95\% Cl 1.22-1.49; 14 studies, 6220 participants), remission ( $R R=1.32,95 \%$ Cl 1.15-1.53; 14 studies, 6220 participants) and depressive symptoms measured using the MontgomeryÅsberg Depression Scale (MADRS) (score range: 0-34; higher score means worse outcome: $\mathrm{MD}=-2.94,95 \% \mathrm{Cl}-4.07$ to $-1.80 ; 14$ studies, 5566 participants). The quality of the evidence was low for response and remission and very low for depressive symptoms. We found no evidence of a difference in total drop-out rates $(\mathrm{RR}=1.05,95 \% \mathrm{Cl} 0.93-1.19 ; 14$ studies, 6220 participants). More participants discontinued vortioxetine than placebo because of adverse effects ( $R R=1.41,95 \%$ Cl 1.09-1.81; 14 studies, 6220 participants) but fewer discontinued because of inefficacy (RR $=0.56,95 \% \mathrm{Cl} 0.34-0.90, P=0.02 ; 14$ studies, 6220 participants). The quality of the evidence for drop-out was moderate. The subgroup and sensitivity analyses did not reveal factors that significantly influenced the results.

In comparison with other antidepressants, very low-quality evidence from eight studies showed no clinically significant difference between vortioxetine and SNRIs as a class for response ( $\mathrm{RR}=0.91,95 \% \mathrm{Cl} 0.82-1.00 ; 3159$ participants) or remission ( $R R=0.89,95 \% \mathrm{Cl} 0.77-1.03 ; 3155$ participants). There was a small difference favouring SNRIs for depressive symptom scores on the MADRS (MD =1.52, 95\% Cl 0.50-2.53; 8 studies, 2807 participants). Very low-quality evidence from eight studies (3159 participants) showed no significant differences between vortioxetine and the SNRIs as a class for total drop-out rates ( $R R=0.89,95 \% \mathrm{Cl} 0.73-1.08)$, drop-out due to adverse events ( $\mathrm{RR}=0.74,95 \% \mathrm{Cl} 0.51-1.08)$ and drop-out due to inefficacy ( $\mathrm{RR}=1.52,95 \% \mathrm{Cl} 0.70-3.30)$

Against individual antidepressants, analyses suggested that vortioxetine may be less effective than duloxetine in terms of response rates (RR $=0.86,95 \% \mathrm{Cl} 0.79-0.94 ; 6$ studies, 2392 participants) and depressive symptoms scores on the MADRS scale (MD =1.99, 95\% Cl 1.15-2.83; 6 studies; 2106 participants). Against venlafaxine, meta-analysis of two studies found no statistically significant differences (response: $\mathrm{RR}=1.03,95 \% \mathrm{Cl}$ 0.85-1.25; 767 participants; depressive symptom scores: $M D=$ $0.02,95 \% \mathrm{Cl}-2.49-2.54 ; 701$ participants). In terms of number of participants reporting at least one adverse effect (tolerability), vortioxetine was better than the SNRls as a class (RR $=0.90$, 95\% Cl 0.86-0.94; 8 studies, 3134 participants) and duloxetine (RR $=0.89,95 \% \mathrm{Cl} 0.84-0.95 ; 6$ studies; 2376 participants). However, the sensitivity analysis casts some doubts on this result, as only two studies used comparable dosing.

We judged none of the studies to have a high risk of bias for any domain, but we rated all studies to have an unclear risk of bias of selective reporting and other biases.

\section{Authors' conclusions}

The place of vortioxetine in the treatment of acute depression is unclear. Our analyses showed vortioxetine may be more effective than placebo in terms of response, remission and depressive symptoms, but the clinical relevance of these effects is uncertain. Furthermore, the quality of evidence to support these findings was generally low. In comparison with SNRIs, we found no advantage for vortioxetine. Vortioxetine was less effective than duloxetine, but fewer people reported adverse effects when treated with vortioxetine compared with duloxetine. However, these findings are uncertain and not well supported by evidence. A major limitation of the current evidence is the lack of comparisons with the SSRIs, which are usually recommended as first-line treatments for acute depression. Studies of direct comparisons with SSRIs are needed to address this gap and may be supplemented by network meta-analyses to define the role of vortioxetine in the treatment of depression. 